\title{
Drug delivery to atherosclerotic plaques using superparamagnetic iron oxide nanoparticles
}

This article was published in the following Dove Press journal:

International Journal of Nanomedicine

\author{
Jasmin Matuszak \\ Barbara Lutz \\ Aleksander Sekita \\ Jan Zaloga \\ Christoph Alexiou \\ Stefan Lyer* \\ Iwona Cicha*
}

Cardiovascular Nanomedicine Unit, Section of Experimental Oncology and Nanomedicine (SEON), Else Kröner-Fresenius-Stiftung-endowed Professorship for Nanomedicine. ENT Department, University Hospital Erlangen, Friedrich-AlexanderUniversität Erlangen-Nürnberg, Erlangen, Germany

*These authors contributed equally to this work
Correspondence: Iwona Cicha Cardiovascular Nanomedicine Unit, Section of Experimental Oncology and Nanomedicine (SEON), Else KrönerFresenius-Stiftung-endowed Professorship for Nanomedicine, ENT Department, University Hospital Erlangen, FriedrichAlexander-Universität Erlangen-

Nürnberg, Glückstr 10a, 91054 Erlangen, Germany

Tel +49 9|3। 8543953

Fax +49 913। 8534282

Email iwona.cicha@uk-erlangen.de
Introduction: Magnetic drug targeting utilizes superparamagnetic iron oxide nanoparticles (SPIONs) to accumulate drugs in specified vasculature regions.

Methods: We produced SPIONs conjugated with dexamethasone phosphate (SPION-DEXA). The efficacy of magnetic drug targeting was investigated in a rabbit model of atherosclerosis induced by balloon injury and high cholesterol diet.

Results: In vitro, SPION-DEXA were well-tolerated by endothelial cells. SPION-DEXA were internalized by human peripheral blood mononuclear cells and induced CD163 expression comparable with the free drug. In vivo, magnetic targeting of SPIONs to abdominal aorta was confirmed by histology. Upon vascular injury followed by high-cholesterol diet, early administration of SPION-DEXA enhanced the inflammatory burden in the plaques. Increased macrophage content and larger intimamedia thickness were observed in animals treated with SPION-DEXA compared with controls. In advanced atherosclerosis, no beneficial effect of local glucocorticoid therapy was detectable.

Conclusion: Magnetic drug targeting represents an efficient platform to deliver drugs to diseased arteries in vivo. However, targeting of vascular injury in the lipid-rich environment using dexamethasone-conjugated SPIONs may cause accelerated inflammatory response.

Keywords: magnetic nanoparticles, magnetic drug targeting, rabbit model of atherosclerosis, dexamethasone, macrophage accumulation

\section{Introduction}

Atherosclerosis represents one of the biggest health care and societal challenges worldwide, ${ }^{1,2}$ but despite intensive efforts, little clinical success has been achieved in terms of targeted drug delivery nanosystems for the disease therapy. ${ }^{3}$ To allow a better control of nanoparticle biodistribution and to enhance their therapeutic efficacy, active targeting is required. In this respect, magnetic nanoparticles constitute an attractive platform for the purpose of medical applications because of their utility both as a diagnostic (contrast) agent and as a drug delivery system. ${ }^{4}$ For these nanoparticles, a promising strategy of drug delivery is based on the so-called "magnetic drug targeting" (MDT). In this approach, superparamagnetic iron oxide nanoparticles (SPIONs) conjugated with drugs are used in combination with an external magnetic field to target the particles to the diseased vasculature regions. This results in increased drug payloads in the target tissue, at the same time reducing their systemic dose and toxicity, as demonstrated by the studies in a rabbit model of cancer, ${ }^{5-7}$ a mouse model of thrombosis, ${ }^{8}$ a mouse model of cardiac ischemia, ${ }^{9}$ and several mouse models of cancer. ${ }^{10-12}$ The existing studies mostly utilized the phenomenon of an enhanced permeability of the microvessel endothelium in cancer and inflammatory diseases, which facilitates the extravasation and retention of nanosized particles. ${ }^{13}$ In contrast, the experimental attempts to magnetically target medium and large vessels have been 
very scarce, ${ }^{14-16}$ and little is known about the feasibility of MDT in atherosclerosis. Our previous studies in an ex vivo artery models suggested that magnetic accumulation of certain types of SPIONs is possible even under arterial flow conditions. ${ }^{17,18}$ Other preclinical studies indicated that SPION-loaded cells can be magnetically targeted to stented carotid arteries in vivo in order to prevent thrombosis and restenosis in a rat model. ${ }^{19}$ By increasing the effective local doses of pharmaceutical agents, MDT may therefore represent a useful tool to improve the plaque stability and reduce inflammatory responses.

Glucocorticoids, potent immunomodulatory agents, represent a key pillar in pharmacotherapy of inflammatory diseases..$^{20}$ Previous studies suggested that glucocorticoid receptor (GR) may mediate the atheroprotective effects of laminar shear stress. ${ }^{21}$ Liposomal prednisolone was furthermore shown to reduce inflammation in a rabbit model of advanced atherosclerosis. ${ }^{22}$

Dexamethasone (DEXA), having a higher degree of GR selectivity (about ten-fold higher than prednisolone) and longer biological half-life (36-54 hours), ${ }^{23}$ is used predominantly to suppress various inflammatory, allergic, and autoimmune disorders. Because of ubiquitous presence of GR in human tissues, the systemic administration of DEXA may result in a variety of adverse side effects, which can be reduced by local or targeted application. In the context of atherosclerosis, local anti-inflammatory therapy with DEXA was previously tested in several clinical studies using DEXA-eluting stents. ${ }^{24}$ The studies that evaluated the clinical outcomes of these stents differed strongly in design and reported inconsistent findings. In some cohorts, no benefits of DEXA were seen over other types of drug-eluting stents, ${ }^{25,26}$ whereas the randomized studies showed lower restenosis and target lesion revascularization rates, as well as reduced major cardiac adverse effects in patients receiving DEXA-eluting stents compared with bare metal stents. ${ }^{27-29}$ Although the interpretation of the existing reports is confounded by the differences in stent material, drug concentrations, and elution rates, the potent antiproliferative effect of DEXA on smooth muscle cells $\mathrm{s}^{30}$ and its anti-inflammatory activity are expected to prevent intimal hyperplasia in response to vascular injury.

Here, we tested the feasibility of MDT to arterial vessels in vivo and the therapeutic efficacy of SPIONs conjugated with dexamethasone phosphate (SPION-DEXA). The SPION formulation used in this study as drug carrier was carefully selected based on the previous in vitro, ex vivo, and in vivo studies, which indicated its good biocompatibility, as well as good magnetic targeting properties. ${ }^{17}$
In order to address the efficacy of targeted inhibition of proinflammatory processes in vascular injury and advanced atherosclerosis, SPION-DEXA were administered and magnetically targeted to the abdominal aorta in the rabbit model of balloon injury and diet-induced atherosclerosis.

\section{Methods}

\section{Reagents and antibodies}

Iron (II) chloride tetrahydrate was from Merck (Darmstadt, Germany). Recombinant human serum albumin (HSA) was purchased from Novozymes Biopharma (Bagsvaerd, Denmark) and dexamethasone-21 phosphate disodium salt from Alfa Aesar (Thermo Fisher, Karlsruhe, Germany). Lauric acid was from Sigma-Aldrich (Munich, Germany). $\mathrm{NaOH}, \mathrm{HCl}(25 \%), \mathrm{NH} 3(25 \%)$, potassium ferrocyanide, and nitric acid $(65 \% \mathrm{w} / \mathrm{w})$ were from Roth (Karlsruhe, Germany). Iron (III) chloride hexahydrate was purchased from Sigma-Aldrich or from Roth. All compounds used were of pharmaceutical (Ph Eur) or highly pure ( $\geq 99 \%$ ) grade and were used without any further purification.

Sodium heparin was obtained from Braun (Melsungen, Germany). Monocyte chemoattractant protein-1 was from tebu-bio (Offenbach, Germany). Endothelial cell growth medium was obtained from PromoCell (Heidelberg, Germany). Nuclear dye Hoechst 33342 was from Life Technologies GmbH (Darmstadt, Germany) and propidium iodide from Sigma. Trichrome stain reagents (acid fuchsin, acridine orange, $1 \%$ molybdophosphoric acid, and light green) were purchased from Merck and hematoxylin from Dako (Hamburg, Germany). Fast Red stain was from Roth. Mouse antihuman CD163 antibody (PE-labeled) and isotype control were from BioLegend (Fell, Germany), and mouse monoclonal antibody against rabbit macrophages (clone RAM-11) was purchased from Dako.

\section{Nanoparticles}

Lauric acid/HSA-coated magnetite nanoparticles (SPION) were synthesized by coprecipitation of iron (II) and iron (III) at a stoichiometric ratio of 1:2 under argon atmosphere at $90^{\circ} \mathrm{C}$, subsequent in situ coating with lauric acid, and formation of an artificial albumin corona using clinically approved HSA formulation, as previously described by Zaloga et al. ${ }^{31}$ Subsequently, control SPIONs were purified by tangential ultrafiltration (molecular weight cutoff $100 \mathrm{kDa}$ ) and, prior to their use in cell culture or in vivo experiments, sterilized by filtration through a $0.22 \mu \mathrm{m}$ filter. Z-averaged hydrodynamic diameter, polydispersity index (PDI), and $\zeta$-potential were determined with a Zetasizer Nano ZS (Malvern Instruments, 
Malvern, UK). To achieve the adsorptive bonding of SPIONDEXA, the coated particles were mixed with the required volume of a sterile solution of $10 \mathrm{mg} / \mathrm{mL}$ DEXA in PBS. The ratio of bound drug was determined as mass/mass ratio of DEXA in the supernatant/DEXA added mass. To assess the drug release kinetics of these particles, dialysis assay was used. For this purpose, $3 \mathrm{~mL}$ SPION-DEXA particles (loaded with $100 \mu \mathrm{g} / \mathrm{mL}$ DEXA phosphate) were placed in an $8 \mathrm{kDa}$ cutoff dialysis bag and incubated in $97 \mathrm{~mL} \mathrm{PBS}$, at $37^{\circ} \mathrm{C}$ in incubation shaker. At specified time points, $3 \mathrm{~mL}$ of the acceptor medium was collected. The samples were concentrated using a C-18 Bond Elut column and redispersion in HPLC mobile phase comprising potassium dihydrogen phosphate (50 mM, pH 7.0) and acetonitrile in a 73/27 (v/v) ratio. The DEXA concentration was determined using HPLC-ultraviolet, with the flow rate maintained at $0.7 \mathrm{~mL} / \mathrm{min}$ and ultraviolet detection at $245 \mathrm{~nm}$. The sample injection volume was $50 \mu \mathrm{L}$ and the column temperature was maintained at $40^{\circ} \mathrm{C}$.

\section{Cells}

Human umbilical vein endothelial cells (HUVECs) were isolated from freshly collected umbilical cords (kindly provided by the Department of Gynecology, University Hospital Erlangen) and cultured as previously described. ${ }^{32}$ For all experiments, HUVECs at passages 1-2 were used.

Peripheral blood mononuclear cells (PBMCs) were isolated from freshly collected human whole blood by density gradient isolation. Blood drawn from healthy donors was anticoagulated with sodium citrate and mixed 2:1 with PBS. The PBS-blood mix ( $30 \mathrm{~mL}$ ) was added on top of $15 \mathrm{~mL}$ of Ficoll and centrifuged at 850 rcf without brake for 20 minutes at room temperature (RT). After removal of the plasma layer, the buffy coat containing PBMCs was collected and washed twice with $50 \mathrm{~mL}$ of PBS ( $650 \mathrm{rcf}, 5$ minutes, RT). The use of human material was approved by the local ethics committee at the University Hospital Erlangen (review numbers 4449 from 11.05.2011 and 335_16 Bc from 22.11.2016) and the donors provided written informed consent.

THP-1 monocytic cells were grown in RPMI 1640 medium supplemented with $2 \mathrm{mmol} / \mathrm{L}$ glutamine, $100 \mathrm{U} / \mathrm{mL}$ penicillin, $100 \mu \mathrm{g} / \mathrm{mL}$ streptomycin, and $10 \%$ fetal calf serum. Viability of cells was $>98 \%$ as estimated by trypan blue exclusion.

\section{In vitro evaluation of cellular effects Real-time cell analysis}

We monitored the effect of the free and nanoparticlebound glucocorticoid, as wells as the control SPIONs on
HUVEC viability using the xCELLigence System (RTCA, SP Analyzer; ACEA Biosciences Inc., San Diego, CA, USA). Microelectrodes localized at the bottom of the wells measured the impedance, which correlates with the viability and attachment strength of cells. Background measurements were performed with $50 \mu \mathrm{L}$ cell-free medium. Afterward, $50 \mu \mathrm{L}$ of HUVEC suspension containing $2 \times 10^{3}$ cells was added. Cell growth was monitored every 10 minutes for a total of 96 hours. At 24 hours after initial seeding, additional $100 \mu \mathrm{L}$ of medium with free DEXA, SPION-DEXA $(0.1,1,10 \mu \mathrm{g} / \mathrm{mL}$ DEXA, or corresponding SPION concentrations: 4 , 40, or $400 \mu \mathrm{g} \mathrm{Fe} / \mathrm{mL}$ ), or control SPIONs was added. The experiments were performed in quadruplicates.

\section{Live-cell microscopy}

HUVECs were seeded at $2 \times 10^{3}$ cells/well in $100 \mu \mathrm{L}$ medium. After 24 hours postinitial seeding, an additional $100 \mu \mathrm{L}$ of medium containing free DEXA, SPION-DEXA, or control SPIONs $(0.1,1,10 \mu \mathrm{g} / \mathrm{mL}$ DEXA, or corresponding SPION concentrations: 4, 40, or $400 \mu \mathrm{g} \mathrm{Fe} / \mathrm{mL}$ ) was added. Cell growth was monitored for a total of 96 hours after initial seeding using the live-cell imager (IncuCyte, FLR Microscope System, Essen BioScience, Ann Arbor, MI, USA). The experiments were performed in hexaplicates.

\section{Endothelial cell migration assay}

Effects of free DEXA, SPION-DEXA, or control SPIONs were assessed using a modified barrier assay based on silicone cell culture inserts placed in 8-well chamber slides (both from ibidi, Munich, Germany). HUVECs were seeded in the two insert wells at a concentration of $3 \times 10^{5}$ cells $/ \mathrm{mL}$. After the adherence time of 4-5 hours, cells were treated with free DEXA ( $1 \mu \mathrm{g} / \mathrm{mL})$, SPION-DEXA $(1 \mu \mathrm{g} / \mathrm{mL}$ DEXA, corresponding to $40 \mu \mathrm{g} \mathrm{Fe} / \mathrm{mL}$ ), or control SPIONs $(40 \mu \mathrm{g} \mathrm{Fe} / \mathrm{mL})$ overnight. The following day, the inserts were removed producing a cell-free gap, and medium containing free DEXA, SPION-DEXA, or control SPIONs was added to respective samples for the next 24 hours. The gap between the two resulting cell monolayers was recorded at the time of insert removal and every hour for the subsequent 24 hours using the IncuCyte FLR System. The size of cell-free areas at the selected time points $(0,12$, and 24 hours) was measured with ImageJ software.

\section{CDI63 staining for analysis of DEXA effectivity}

The ability of free and SPION-bound DEXA to stimulate human PBMCs was investigated by measuring CD163 
expression, which is induced by glucocorticoids. ${ }^{33}$ The isolated PBMCs were seeded at $1 \times 10^{6}$ cells per well in $1 \mathrm{~mL}$ medium, in a 12-well plate. The cells were incubated with free DEXA or SPION-DEXA at a concentration of $1 \mu \mathrm{g} / \mathrm{mL}$ DEXA. The control cells were treated either with medium only or with control SPIONs (without DEXA) at iron concentrations corresponding to SPION-DEXA. After 48 hours, PBMCs were divided into two batches, one for CD163 analysis and the second for iron content measurement. To assess the fluorescence intensity of CD163, samples were stained with Hoechst to gate for healthy nucleated cells. CD163 expression in PBMCs stained with anti-CD163 antibody (1:100), or isotype control, was measured with Beckman Coulter flow cytometer.

\section{Iron content measurement}

PBMCs were analyzed for the particle uptake after 48 hours by measuring intracellular iron with microwave plasma atomic emission spectroscopy (MP-AES, 4200 device; Agilent Technologies, Santa Clara, CA, USA). Briefly, cell numbers were determined in each sample, followed by cell pelleting by centrifugation. The dry pellets were dissolved in $50 \mu \mathrm{L}$ of $65 \%$ nitric acid for 10 minutes at $95^{\circ} \mathrm{C}$ and $600 \mathrm{rpm}$ in a thermomixer. Afterward, $450 \mu \mathrm{L}$ of distilled water was added and the emission spectra of the samples were analyzed and compared with the standard curves.

\section{Chemotaxis assay}

The effect of particles on monocytic cell migration was evaluated in a 96-well ChemoTx plate (NeuroProbe, Gaithersburg, MD, USA). THP-1 monocytic cells were incubated with $0.1,1$, or $10 \mu \mathrm{g} / \mathrm{mL}$ of free DEXA, SPION-DEXA, or control SPIONs (SPION concentration corresponding to SPION-DEXA amount) for 2 hours at $37^{\circ} \mathrm{C}$. The lower wells were filled with serum-free RPMI 1640, and monocyte chemoattractant protein (MCP-1; $50 \mathrm{ng} / \mathrm{mL}$ ) was used as a positive control. Free drug or nanoparticle-treated monocytic cells $\left(50 \mu \mathrm{L}\right.$ at $1 \times 10^{6}$ cells $\left./ \mathrm{mL}\right)$ were pipetted on top of the filter frame with $5 \mu \mathrm{m}$ pores. After 1 hour of incubation at $37^{\circ} \mathrm{C}$, migrated nonadherent cells from the lower wells were fixed and counted using flow cytometry. The mean number of migrated cells in the positive control (MCP-1 stimulated) samples was set as $100 \%$.

\section{Animals}

A rabbit model of balloon injury- and Western diet-induced atherosclerosis was used to test the MDT efficacy. The study protocol was approved by the responsible authority (Regierung von Mittelfranken in Ansbach, permission 54-2532.1-21/11) implementing the German Animal Welfare
Act. Animal housing and care taking were in agreement with the directive 2010/63/EU.

Twenty-three New Zealand White rabbits (weighing 3.0-4.0 kg; age 3-4 months; Charles River, France) were included in the study. General anesthesia was induced and maintained using ketamine $(25 \mathrm{mg} / \mathrm{kg})$ and xylazine (2.5 mg/kg). A 4-French sheath was inserted into the left common carotid artery exposed through a midline neck incision. Heparin (500 U) was administered intravenously prior to MDT and for 7 days after the procedure in the form of subcutaneous injection. A 4-French catheter was advanced into the descending aorta, and an angiogram of the distal aorta was performed, followed by the balloon injury of lower abdominal aorta. Upon application of contrast agent, 3D images were acquired with the Siemens Artis Angiography System (Siemens, Erlangen, Germany).

The following day, the high-cholesterol diet was initiated, whereby the animals were fed an atherogenic diet consisting of $1 \%$ cholesterol for 5 weeks. After that time, the diet was switched to a normal chow for the remainder of the study. As a baseline, nanoparticle-untreated control group was included. In these $n=5$ control animals, atherosclerosis was induced by ballooning followed by atherogenic diet as described above. Seven weeks after balloon injury, the animals were sacrificed and the aortas dissected for further histochemical analyses.

\section{Nanoparticle administration}

To confirm nanoparticle accumulation at the region of aortic injury, the balloon injury of lower abdominal aorta was induced in $\mathrm{n}=2$ animals, followed by an immediate intra-arterial infusion of control SPIONs under external magnetic field (custom-built electromagnet; Siemens, Erlangen, Germany). A marking net with a reference frame visible on the scans was used to achieve the correct positioning of the magnet tip at the target region. The magnetic field gradient at the tip of the pole shoe was set for $72 \mathrm{~T} / \mathrm{m}$ (which corresponded to about $40 \mathrm{~T} / \mathrm{m}$ field gradient at the targeted region) and was maintained for 45 minutes. Nanoparticles $(3 \mathrm{~mL})$ were administered in cycles of balloon opening and closing in steps of $0.2 \mathrm{~mL}$ per minute. Subsequently, the animals were euthanized with pentobarbital overdose and the aortas dissected for histochemical analysis of iron content.

As the immunization may occur in rabbits upon repeated administration of $\mathrm{HSA}$, we have chosen to use a single application of SPIONs. A total of 16 atherosclerotic New Zealand White rabbits received therapeutic nanoparticle administration according to the following protocol:

1. Early treatment: Following the balloon injury on lower abdominal aorta, the animals received the immediate 
infusion of control SPIONs (total volume $3 \mathrm{~mL}, \mathrm{n}=5$ ) or SPION-DEXA (total volume $3 \mathrm{~mL}$, total DEXA concentration $300 \mu \mathrm{g}, \mathrm{n}=4$ ) under external magnetic field. Subsequently, the animals received high-cholesterol diet for 5 weeks, followed by normal diet for 2 weeks (recovery period). After the angiographic visualization of the plaques, the animals were sacrificed and the aortas dissected for further analyses. This protocol was used to assess the early anti-inflammatory and atheropreventive activity of SPION-DEXA.

2. Late treatment: Following the balloon injury on lower abdominal aorta, the animals received high-cholesterol diet for 5 weeks, followed by normal diet for 2 weeks (recovery period). After the angiographic visualization of the developed plaques, the animals received the infusion of control SPIONs (total volume $3 \mathrm{~mL}, \mathrm{n}=4$ ) or SPIONDEXA (total volume $3 \mathrm{~mL}$, total DEXA concentration $300 \mu \mathrm{g}, \mathrm{n}=3$ ) under external magnetic field. Four weeks after nanoparticle administration, the animals were sacrificed and the aortas dissected for further histochemical analyses. This protocol was used to investigate the effects of SPION-DEXA on advanced lesions.

\section{Histological and immunohistochemical staining}

Aortic specimens, containing the entire intima, media, and a part of the adventitia, were placed in embedding cassettes and fixed in 4\% formaldehyde solution in PBS buffer for 5 days. The aortas were dehydrated in an ascending isopropanol. After macroscopic inspection, the specimens (total length of $\sim 4-5 \mathrm{~cm}$ ) were cut into five equal segments labeled 1-5, starting at the aortic bifurcation and embedded in paraffin. Microtome sections of $5 \mu \mathrm{m}$ thickness were obtained proximally and placed on silane-coated slides. The sections were dewaxed in xylene, rehydrated in ethanol, and washed with Tris-buffered saline containing $0.1 \%$ Tween 20 . The sections were stained as described before using Dako catalyzed signal amplification System ${ }^{\mathrm{TM}}$ with diaminobenzidine as a chromogene, followed by counterstaining with hematoxylin. Mouse monoclonal antibody against rabbit macrophages (RAM-11, 1:50 dilution) was used to detect plaque macrophages. Tissue specimens were mounted using a durable medium (Aquatex; Merck, Darmstadt, Germany).

To characterize the arterial specimens, vessel morphology was analyzed using Crossman's trichrome stain for muscle and collagen, whereby the collagen fibers were stained green, the nuclei were stained black, and the cytoplasm and muscle tissue were stained red. Briefly, samples were stained with hematoxylin for 10 minutes, followed by rinsing with tap water for 10 minutes. Afterward, the samples were stained for $\sim 1$ minute with a mixture of acid fuchsin and acridine orange and subsequently washed with distilled water. The resulting red staining was differentiated with $1 \%$ molybdophosphoric acid until the connective tissue was decolorized. After washing with distilled water, staining with light green was performed for 5 minutes with subsequent washing. Following dehydration in isopropanol gradient and xylene, mounting medium was used to preserve the staining (Roti-Histo Kit; Roth).

\section{Tissue iron content by histology}

The sections were stained with Prussian blue to assess the presence of SPIONs. The paraffin-embedded serial sections of $5 \mu \mathrm{m}$ thickness were dewaxed in xylene, rehydrated in ethanol, and immersed in 1:1 solution of hydrochloric acid (2\%) and potassium ferrocyanide (2\%) for 30 minutes at RT. Nuclei of the cells and cytoplasm were counterstained with Fast Red stain, followed by rinsing with distilled water and subsequent dehydration in isopropanol gradient and xylene and mounting (Roti-Histo Kit).

\section{Plaque morphometry and image analysis}

Digital images of the plaques were obtained using AxioObserver.Z1 microscope (Zeiss, Jena, Germany). Plaque area and thickness were measured using ImageJ software. Plaque area was calculated by measuring the surface delimited by the internal elastic lamina and distracting the surface of the lumen. The results were normalized to total vessel cross-sectional area for each arterial section to eliminate variations due solely to vessel size. The minimum fibrous cap thickness at each plaque section and the media thickness at the thickest region of the plaque were measured for each section. Intima-media thickness (IMT) was calculated.

To analyze the macrophage load in the plaques, the color threshold for immunostained cells was manually adjusted in the images until the computerized detection matched the visual interpretation (MetaVue Software; Molecular Devices LLC, San Jose, CA, USA). The numbers of immunoreactive cells were additionally digitally counted (NIS-Elements; Nikon Instruments, Düsseldorf, Germany) in the representative areas of $0.2 \mathrm{~mm}^{2}$ in the plaque shoulders, lipid core, and fibrous cap. Analyses were carried out by two independent observers (BL, IC). The intra- and interobserver variability was lower than $10 \%$.

\section{Statistical analysis}

Data were expressed as median \pm standard error of the mean (SEM), unless stated otherwise. $P<0.05$ was considered 
statistically significant. Analyses were carried out using SigmaStat $^{\circledR}$ software. For in vitro comparison of multiple treatment groups, one-way ANOVA was used. The unpaired Student's $t$-test (in samples with normal distribution) or Mann-Whitney rank sum test were used to compare the effects of treatment between the in vivo study groups.

\section{Results}

\section{Nanoparticle characteristics and drug release}

The produced control SPIONs had hydrodynamic diameter of $63.9 \pm 1.9 \mathrm{~nm}, \zeta$-potential of $-21.2 \mathrm{mV}$, and PDI of 0.32 . SPION-DEXA had a slightly smaller hydrodynamic diameter $(62.9 \pm 2 \mathrm{~nm})$, slightly more negative charge ( $\zeta$-potential of $-27.2 \mathrm{mV}$ ), and PDI of 0.33. After the ultrafiltration, the amount of bound DEXA was $79.7 \% \pm 0.3 \%$ (w/w). To assess the drug release kinetics of these particles, dialysis assay was used. The release curve of the DEXA from SPION-DEXA is shown in Figure 1. Accordingly, about $45 \%$ of the loaded drug was released during the first 24 hours of incubation. It must be noted, however, that this type of release kinetics may be beneficial to the acute aortic injury scenario (as opposed to slow-release drug delivery systems, which are required for effective cancer therapy), where the recruitment of circulating platelets occurs within seconds and of monocytes within 3-5 minutes after the injury.

\section{Effects of SPION-DEXA on endothelial cell viability and migration}

In vitro analysis of biocompatibility of SPION-DEXA with HUVECs was performed by real-time cell analysis and livecell microscopy. As shown in Figure 2A and B, there was

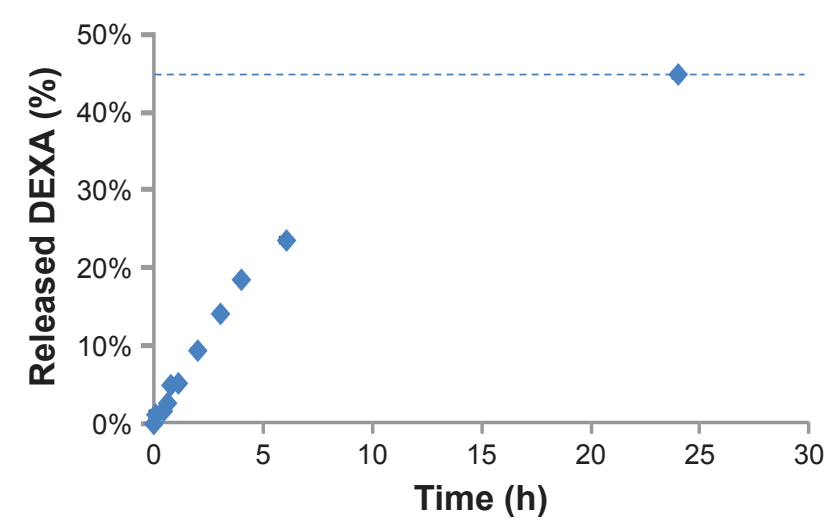

Figure I The release curve of the DEXA from SPION-DEXA.

Notes: Percentage of released DEXA phosphate is shown. Dashed line indicates the amount of drug released after 24 hours.

Abbreviations: DEXA, dexamethasone; SPION-DEXA, SPIONs conjugated with dexamethasone phosphate. no significant effect of SPION-DEXA on the viability of HUVECs in comparison with the untreated control or with free DEXA. Solely at the highest concentrations of SPIONDEXA or control SPIONs ( $400 \mu \mathrm{g} \mathrm{Fe} / \mathrm{mL})$, slight reduction in cell index after 72 hours incubation was observed when compared with untreated samples. However, as the cell confluence evaluated by live-cell microscopy was unchanged at this time point (Figure 2B), it is assumed that the decrease in cell index was related rather to cell shape change and/or weaker cell attachment than to cell death. We further assessed the effect of SPION-DEXA ( $1 \mu \mathrm{g} / \mathrm{mL}$ DEXA, corresponding to $40 \mu \mathrm{g} \mathrm{Fe} / \mathrm{mL}$ ) and the respective controls on endothelial migration. Compared with untreated HUVECs, a significant reduction of migratory ability at 24 hours was observed in both SPION-DEXA and SPION-treated samples (Figure 2C). Since free DEXA $(1 \mu \mathrm{g} / \mathrm{mL})$ did not inhibit endothelial migration, the observed effect is likely related to SPION uptake by HUVECs.

\section{In vitro effects of SPION-DEXA on monocytic cells}

Preincubation of PBMCs with either free DEXA $(1 \mu \mathrm{g} / \mathrm{mL})$ or SPION-DEXA ( $1 \mu \mathrm{g} / \mathrm{mL}$ DEXA, corresponding to $40 \mu \mathrm{g} \mathrm{Fe} / \mathrm{mL}$ ) for 48 hours caused a comparable and significant increase in CD163 expression. In comparison with untreated and control SPION-treated cells, CD163 levels were increased 7.5-fold by DEXA and 8.5-fold by SPIONDEXA (Figure 3A and B), whereby the difference between free DEXA and SPION-DEXA effects was not significant. The measurement of iron load per cell confirmed SPION uptake (Figure 3C). The iron concentrations in PBMCs treated with SPION and SPION-DEXA for 48 hours were $>10$-fold higher than those in untreated and free DEXA-treated cells. There was no significant difference in the particle internalization between SPION-DEXA and SPION-treated PBMCs, indicating that the presence of bound drug does not hinder the particle uptake. The internalization and clearance of SPIONs were also investigated in THP-1 cells (Figure S1), showing similar level of uptake and a gradual decrease in cellular iron concentration within 72 hours postloading.

To further evaluate the effects of SPION-DEXA on monocytic cell chemotaxis, THP-1 cells were incubated with free DEXA, SPION-DEXA, or control SPIONs for 2 hours, followed by 1 hour of migration toward MCP-1. As shown in Figure 3D, free DEXA $(0.1-10 \mu \mathrm{g} / \mathrm{mL})$ had no significant effect on THP-1 chemotaxis to MCP-1. In samples treated with control SPIONs, a biphasic effect was detected, as also observed previously in other iron oxide-based particles. At 



Figure 2 Effects of SPION-DEXA on endothelial cell viability and migration.

Notes: (A) Real-time cell analysis and (B) live-cell microscopy. HUVECs were treated with DEXA, SPION-DEXA, or SPIONs alone for up to 72 hours. Negative controls represent untreated cells. Cell index is displayed as $x$-fold of untreated controls. Data are expressed as mean \pm SEM; $n=3$. (C) Endothelial cell migration. HUVECs were pretreated with free DEXA (I $\mu \mathrm{g} / \mathrm{mL})$, SPION-DEXA (I $\mu \mathrm{g} / \mathrm{mL}$ DEXA, corresponding to $40 \mu \mathrm{g} \mathrm{Fe} / \mathrm{mL})$, or SPIONs (40 $\mu \mathrm{g} \mathrm{Fe} / \mathrm{mL})$ overnight. A gap between two cell layers was created using a cell culture insert. After removal of the insert, cell migration was monitored for 24 hours. Analysis was performed with Imagej. Data are expressed as mean $\pm \mathrm{SEM} ; * * \mathrm{P}<0.0$ I vs untreated control (one-way ANOVA); $\mathrm{n}=3$.

Abbreviations: DEXA, dexamethasone; HUVECs, human umbilical vein endothelial cells; SEM, standard error of the mean; SPION-DEXA, SPIONs conjugated with dexamethasone phosphate. 
A

Control

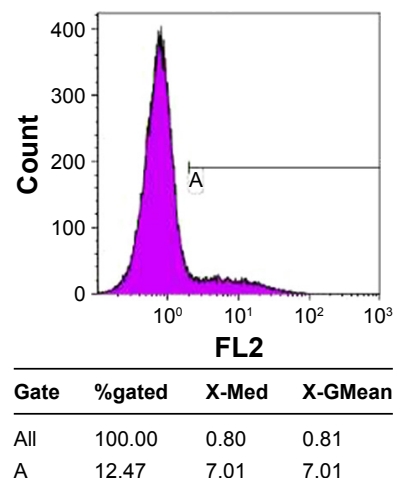

DEXA

(1 $\mu \mathrm{g} / \mathrm{mL})$

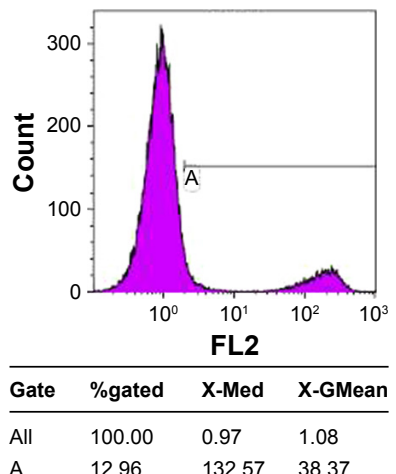

SPION-DEXA

$(1 \mu \mathrm{g} / \mathrm{mL})$

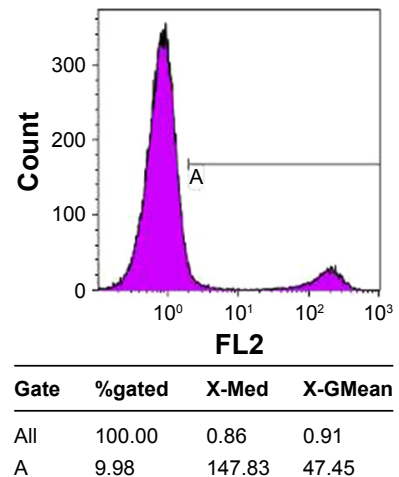

SPION

$(40 \mu \mathrm{g} \mathrm{Fe} / \mathrm{mL})$

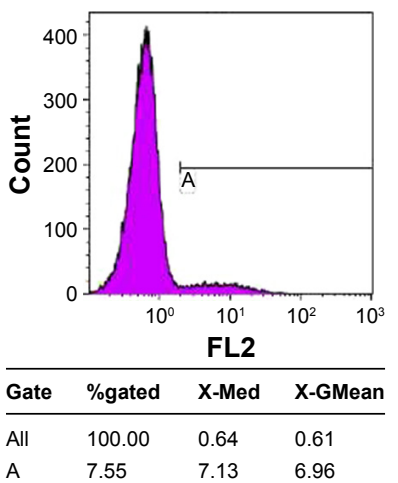

B

CD163 expression

C

Particle uptake in PBMCs
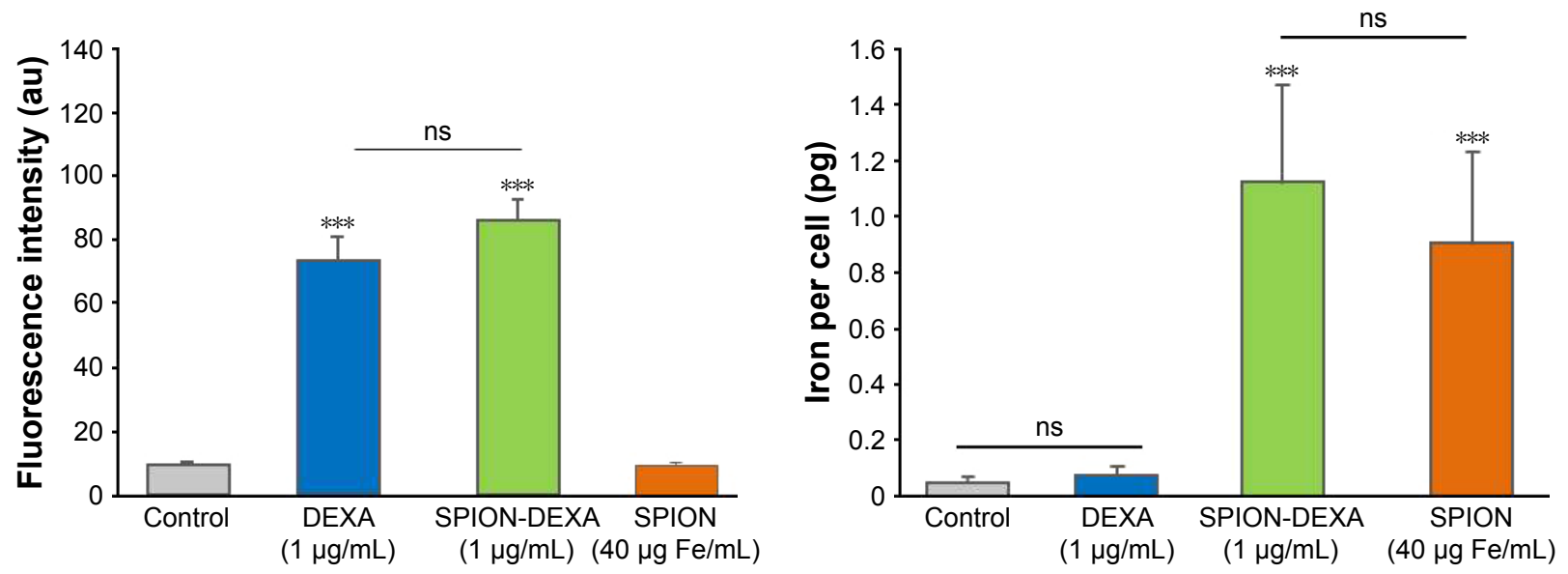

D

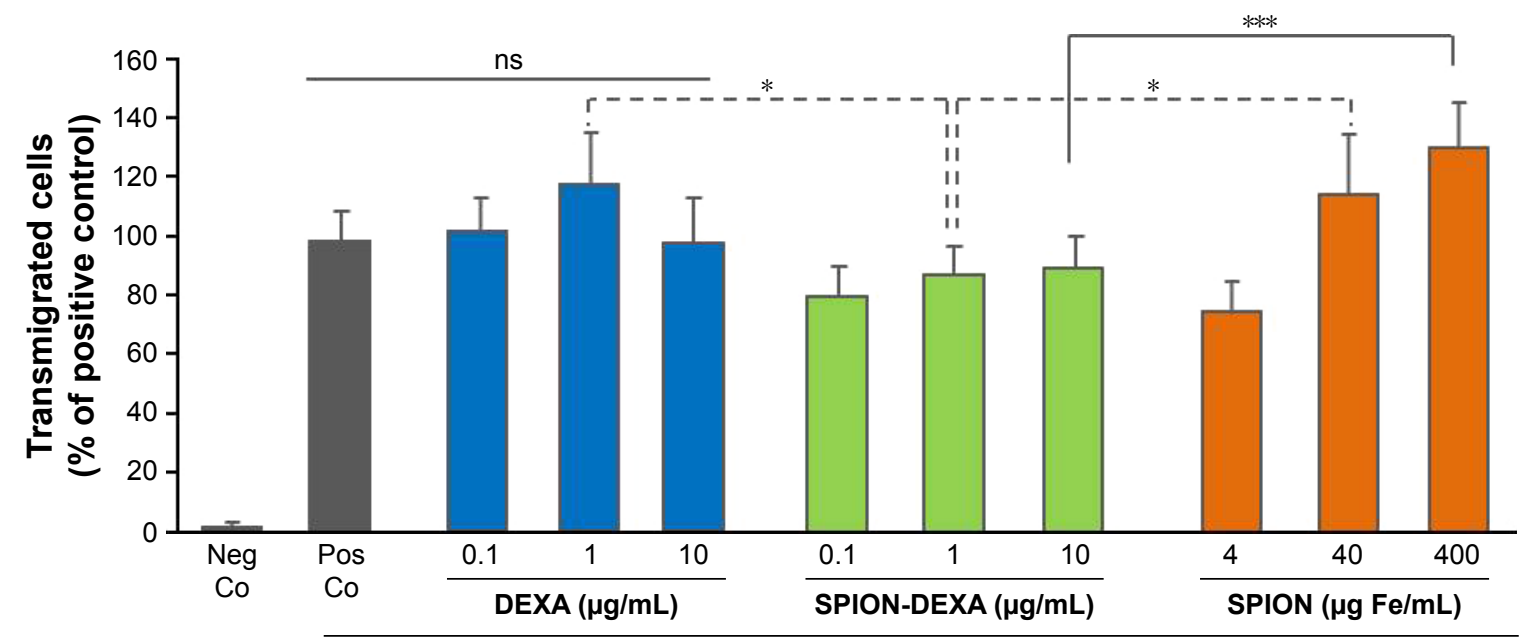

MCP-1 (50 ng/mL)

Figure 3 In vitro effects of SPION-DEXA on monocytic cells.

Notes: (A) Flow cytometric analysis of CDI63 expression in PBMCs treated with free DEXA (I $\mu \mathrm{g} / \mathrm{mL})$, SPION-DEXA (I $\mu g / \mathrm{mL}$ DEXA, corresponding to $40 \mu \mathrm{g}$ Fe/mL), or control SPIONs for 48 hours. (B) Fluorescence intensity quantification. Data are expressed as mean $\pm \mathrm{SEM}$. $* * * P<0.00 \mathrm{I}$ vs untreated control (signed rank test); $\mathrm{n}=3$. (C) SPION uptake by PBMCs. Data are expressed as mean \pm SEM. **** $<0.00$ I vs untreated control (signed rank test); $n=3$. (D) THP-I chemotaxis toward MCP-I was quantified after treatment with DEXA, SPION-DEXA, or SPIONs alone for 2 hours. Nanoparticle-untreated positive control values (with MCP-I) were set to I00\%. Data are expressed as mean $\pm S E M$. $* P<0.05 ; * * * P<0.00$ I vs corresponding concentrations of control SPIONs (signed rank test); $n=3$.

Abbreviations: DEXA, dexamethasone; MCP-I, monocyte chemoattractant protein-I; ns, not significant; Neg Co, negative control; PBMCs, peripheral blood mononuclear cells; Pos Co, positive control; SEM, standard error of the mean; SPION-DEXA, SPIONs conjugated with dexamethasone phosphate. 
low SPION concentrations ( $4 \mu \mathrm{g} \mathrm{Fe} / \mathrm{mL}$ ), chemotaxis was reduced, whereas at high concentrations $(400 \mu \mathrm{g} \mathrm{Fe} / \mathrm{mL})$, it was significantly increased. At the corresponding iron concentrations, SPION-DEXA treatment prevented the increase in THP-1 migration, likely due to the presence of glucocorticoid on the particles.

\section{Magnetic targeting efficacy}

The feasibility of MDT to abdominal aorta was tested by targeting SPIONs to the injured aortic region directly after the ballooning. The setup of the magnetic field generation including placement of the magnetic tip is shown in
Figure 4A. Following the magnetic targeting of control SPIONs, the aortas were excised (Figure 4B) and after histological sample preparation, analyzed for iron content. Prussian blue staining confirmed the presence of iron in the targeted region, but not at the more distal aortic bifurcation region (Figure $4 \mathrm{C}$ and $\mathrm{D}$ ), indicating that it is possible to accumulate the intra-arterially administered SPIONs at the target region of arterial wall in vivo. As expected, the targeting occurs laterally using this setup, due to the placement of magnetic tip on top of the abdominal aorta during SPION administration, which results in iron accumulation on one side of the aorta only.
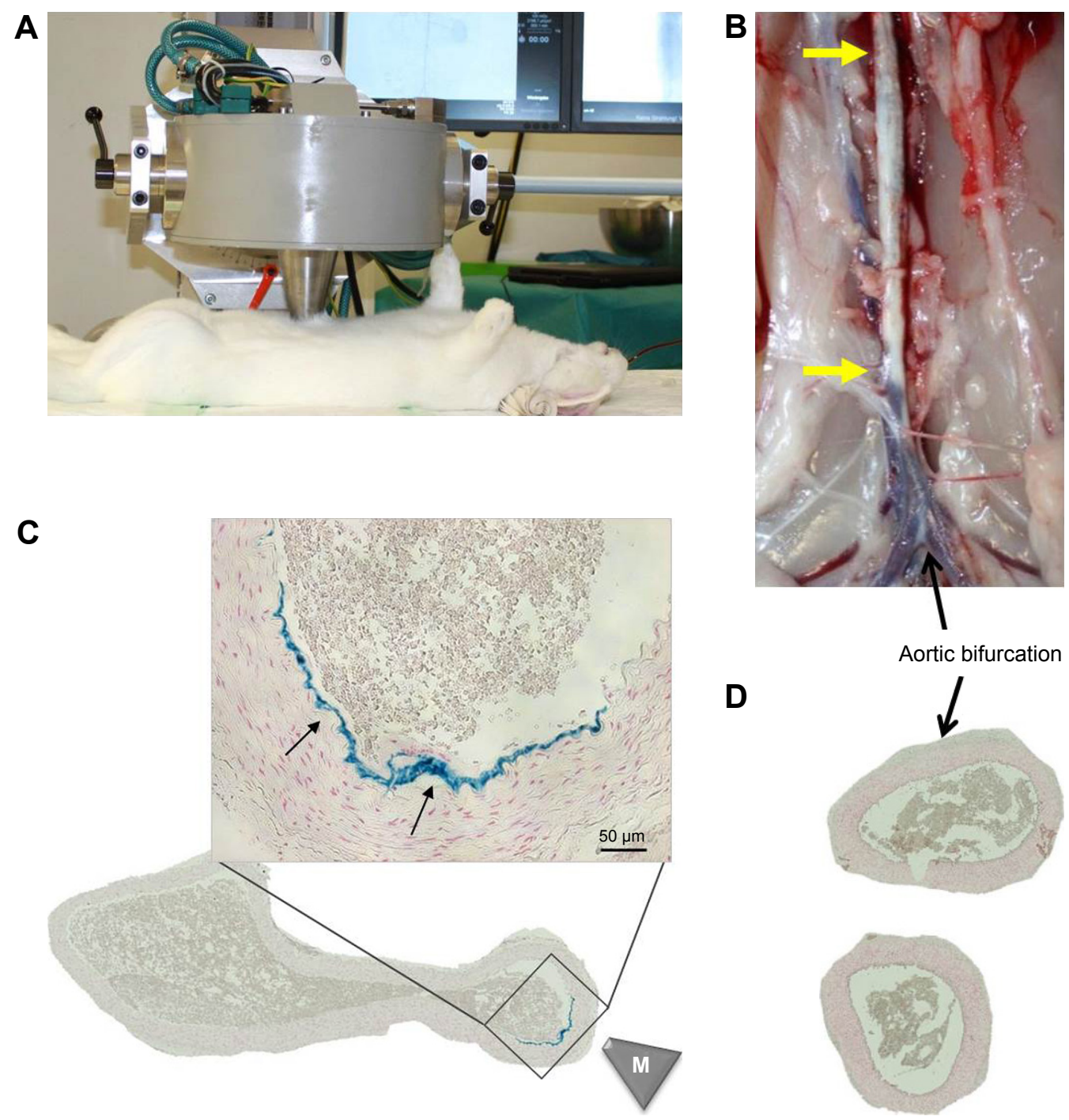

D

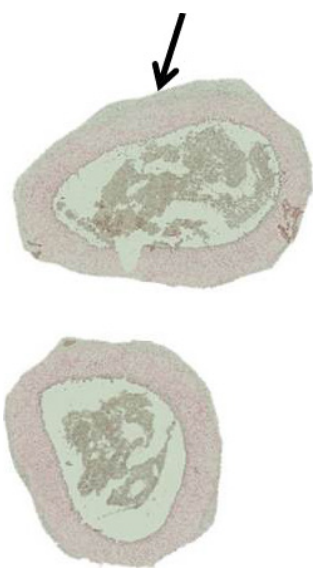

Figure 4 Magnetic targeting setup and efficacy.

Notes: SPIONs were magnetically targeted to the injured aortic region directly after the ballooning. (A) Experimental setup and placement of magnetic tip are shown. (B) Following the magnet exposure for 30 minutes, the animals were sacrificed and the excised aorta analyzed histologically. Atherosclerotic plaque is visible as indicated by yellow arrows. (C) The presence of iron in the targeted region was visualized by Prussian blue staining (arrows). Scale bar: $50 \mu \mathrm{m}$. (D) No iron accumulation was detected in aortic bifurcation region by histology. The overview images of the artery cross-sections were taken at $\times 10$ objective magnification. $M$ indicates the tip of the magnet. Abbreviations: SPIONs, superparamagnetic iron oxide nanoparticles. 


\section{Effects of early SPION-DEXA administration on plaque size and macrophage load}

To assess an anti-inflammatory effect of local glucocorticoid therapy under the conditions of endothelial injury followed by high-cholesterol diet, animals received intra-arterial administration of SPION-DEXA or control SPIONs immediately after the injury, followed by atherogenic diet. Nanoparticle-untreated control group of atherosclerotic animals was included as a baseline control. The normalized surface of the plaques and the minimum fibrous cap thickness did not differ between animals treated with SPION-DEXA and the control-SPION group (Table 1). Compared with control SPION-treated group, a tendency for increased maximum thickness of intima (Crossman's trichrome staining) and, consequently, for increased IMT was observed both in SPION-DEXA early treatment group and in untreated control animals (Figure 5A-C). Contrary to our hypothesis, the local administration of SPION-DEXA enhanced rather than reducing the inflammatory burden in the plaques. The histochemical analyses performed on the excised abdominal plaques showed an increased macrophage content (RAM-11 staining) in animals treated with SPION-DEXA compared with control SPION-administered group (Figures 5D and S2). Furthermore, compared with nanoparticle-untreated control group, early treatment with SPION-DEXA resulted in significantly increased macrophage load (mean $25 \%$ vs $44 \%$; median $29 \%$ vs $42 \% ; P=0.025$ ). There was no difference in macrophage content between untreated animals and control-SPION early treatment group.

Prussian blue staining was negative in all SPION-treated animals, indicating the complete clearance of iron oxide particles from the aortic wall within 7 weeks postadministration (not shown).

\section{Effects of late SPION-DEXA administration on plaque size and macrophage load}

To investigate the effects of SPION-DEXA administration on advanced lesions, animals underwent balloon injury followed by atherogenic diet for 5 weeks and normal diet for 2 weeks. Subsequently, intra-arterial administration of SPION-DEXA or control SPIONs under angiographic guidance was performed, followed by additional 4 weeks of normal diet.

In histological analyses of the plaques at their thickest regions, no differences were observed in the normalized surface of the plaques, minimum fibrous cap thickness, and maximum thickness of intima or IMT between late treatment controls and SPION-DEXA-treated animals (Table 2). Additionally, the local administration of SPIONDEXA at advanced lesions did not significantly affect the macrophage load compared with control SPION-treated animals (Figure 6). Prussian blue staining was negative in all animals, indicating the complete clearance of iron oxide particles from the aortic wall within 4 weeks postadministration (not shown).

We subsequently performed another set of analyses to compare the effects of early vs late treatment with SPIONDEXA. Interestingly, despite negligible differences in plaque size or IMT, a tendency toward stronger fibrous cap thinning ( 32.7 vs $51.4 \mu \mathrm{m} ; P=0.10)$ and significantly increased macrophage-positive surface $(42 \%$ vs $24 \% ; P=0.011)$ was observed in early treatment group compared with animals that received late MDT with SPION-DEXA. In animals treated with control SPIONs, no such differences in macrophage load were observed, although early treatment group had more stable features, including lower normalized plaque surface (66\% vs 75\%), smaller maximum intima thickness (422 vs $540 \mu \mathrm{m}$ ), and smaller IMT (2.78 vs 5.58) compared with late treatment control group.

Table I Plaque morphometry and macrophage burden in animals receiving early administration of SPION-DEXA and control SPIONs

\begin{tabular}{|c|c|c|c|}
\hline & Untreated control & $\begin{array}{l}\text { Early treatment } \\
\text { control SPION }\end{array}$ & $\begin{array}{l}\text { Early treatment } \\
\text { SPION-DEXA }\end{array}$ \\
\hline Plaque area $\left(\mathrm{mm}^{2}\right)$ & $2.37(2.39 \pm 0.6)$ & $1.54(1.63 \pm 0.3)$ & $1.76(1.63 \pm 0.1)$ \\
\hline Normalized plaque area (\%) & $67.8(61.7 \pm 6.2)$ & $66.2(63.3 \pm 5.2)$ & $65.5(66.6 \pm 3.3)$ \\
\hline Maximum plaque thickness $(\mu \mathrm{m})$ & $515.6(493.7 \pm|2| .6)$ & $422.4(445.6 \pm 78.2)$ & $527.8(497.7 \pm 57.3)$ \\
\hline Media thickness $(\mu \mathrm{m})$ & 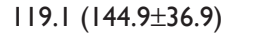 & 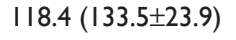 & $110.8(112.3 \pm 7.8)$ \\
\hline IMT & $5.07(4.3 \pm 1.2)$ & $2.78(3.65 \pm 0.8)$ & $4.42(4.40 \pm 0.3)$ \\
\hline Minimum FC thickness $(\mu \mathrm{m})$ & $29.3(34.9 \pm 12.4)$ & $38.0(42.4 \pm 6.8)$ & $32.7(36.3 \pm 4.5)$ \\
\hline Macrophage area (\%) & $29.5(25.7 \pm 4.8)$ & $29.8(30.2 \pm 4.4)$ & $42.3(44.7 \pm 4.5)^{*, \#}$ \\
\hline
\end{tabular}

Notes: Data are expressed as median (mean \pm SEM). ${ }^{*} P=0.025$ vs nanoparticle-untreated control; ${ }^{*} P=0.0285$ vs control SPION-receiving group, one-tailed $t$-test.

Abbreviations: FC, fibrous cap; IMT, intima-media thickness; SEM, standard error of the mean; SPIONs, superparamagnetic iron oxide nanoparticles; SPION-DEXA, SPIONs conjugated with dexamethasone phosphate. 
A

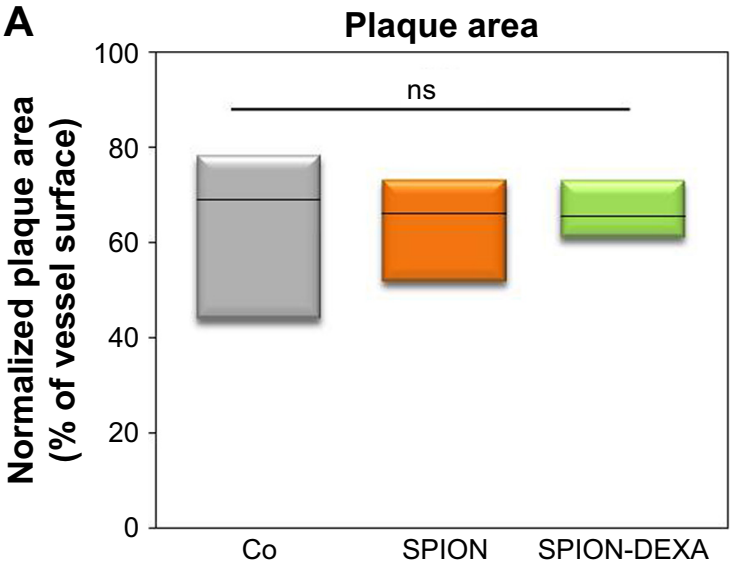

C

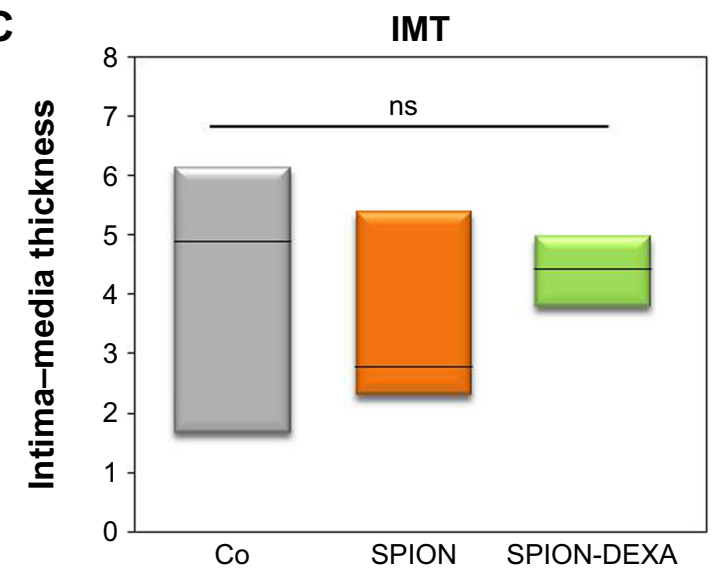

B

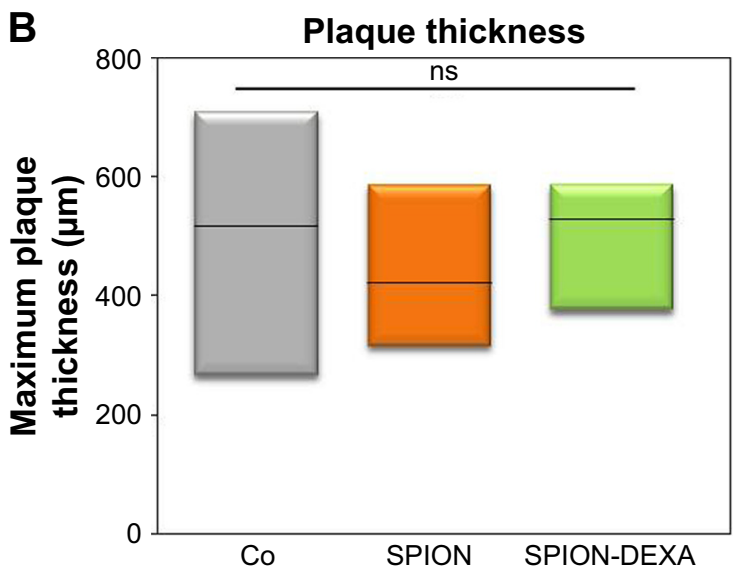

D Macrophage load

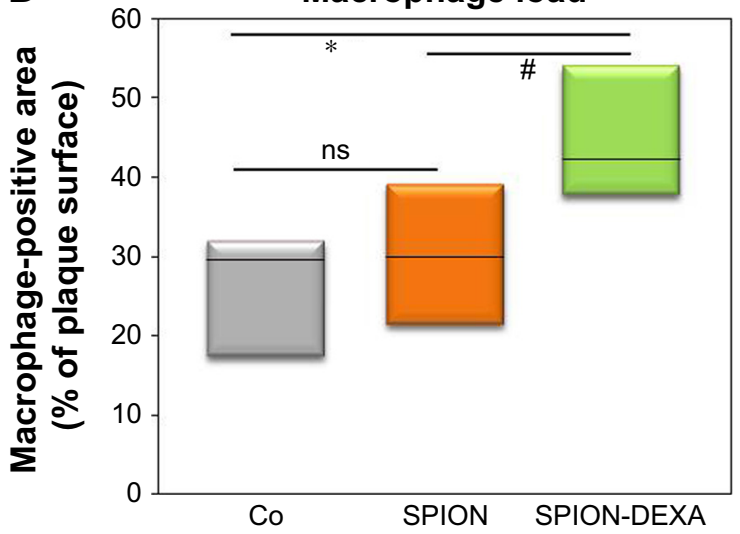

Figure 5 In vivo effects of early MDT with SPION-DEXA.

Notes: Following the balloon injury, the animals were intra-arterially administered either control SPION ( $n=5)$ or SPION-DEXA ( $n=4)$ under external magnetic field. Control group animals $(\mathrm{Co})$ did not receive any treatment with nanoparticles. After 5 weeks of high-cholesterol diet and normal diet for 2 weeks, animals were sacrificed and the excised aortas analyzed histochemically. (A) Normalized plaque area; (B) maximum thickness of intima (measured on Crossman's trichrome stained sections); and (C) the calculated IMT are shown; (D) Macrophage-positive area (RAM-II staining) in animals receiving early administration of control SPION or SPION-DEXA. Graphs show median, 25 th and 75 th percentile. ${ }^{*} P=0.025$ vs nanoparticle-untreated control; ${ }^{*} P=0.0285$ (one-tailed $t$-test), $P=0.057$ (two-tailed $t$-test) vs control $S P I O N$ group.

Abbreviations: IMT, intima-media thickness; MDT, magnetic drug targeting; ns, not significant; SPIONs, superparamagnetic iron oxide nanoparticles; SPION-DEXA, SPIONs conjugated with dexamethasone phosphate.

\section{Discussion}

The aim of the present study was to test the feasibility of MDT to large arterial vessels in vivo. For this purpose, we selected lauric acid/albumin-coated SPIONs as a carrier. These particles have a good biocompatibility profile, as well as good colloidal and blood stability, ${ }^{31}$ and were successfully targeted to the specific artery region ex vivo under external magnetic field gradient. ${ }^{17}$ To our knowledge, this is the first study to demonstrate the possibility of magnetic targeting of SPIONs at the wall of a large artery in a living rabbit. The histological analyses showed the accumulation of iron at the targeted regions, which confirmed our previous ex vivo data, and indicated that magnetic accumulation of this type of SPIONs is possible, at least in superficial and/or easily accessible arteries in vivo. This finding opens the road for further investigations aiming both to improve targeting efficacy and to develop the potential
MDT-based approaches to therapy of arterial injuries and/or atherosclerotic plaques.

However, whereas MDT constitutes the right approach to targeting vascular disorders, DEXA seems to represent a wrong drug in this context. We used DEXA-loaded SPIONs under the assumption that it will diminish the inflammatory process in atherogenesis, as inhibition of atherosclerosis in cholesterol-fed rabbits by systemic administration of antiinflammatory steroids was demonstrated $>60$ years ago. ${ }^{34}$ Subsequent studies showed that also DEXA suppressed the development of atherosclerosis and reduced both macrophage and $\mathrm{T}$ lymphocyte content in the plaques of cholesterolfed New Zealand White rabbits. ${ }^{35}$ Based on these existing reports, we expected that local DEXA therapy will alleviate the inflammatory activation and plaque formation induced by ballooning. Contrary to our hypothesis, under the conditions of acute endothelial injury followed by atherogenic 
Table 2 Plaque morphometry and macrophage burden in animals receiving late administration of SPION-DEXA and control SPIONs

\begin{tabular}{|l|l|l|l|}
\hline & Untreated control & $\begin{array}{l}\text { Late treatment } \\
\text { control SPION }\end{array}$ & $\begin{array}{l}\text { Late treatment } \\
\text { SPION-DEXA }\end{array}$ \\
\hline Plaque area $\left(\mathrm{mm}^{2}\right)$ & $2.37(2.39 \pm 0.6)$ & $2.87(2.53 \pm 0.4)$ & $1.67(2.23 \pm 0.9)$ \\
\hline Normalized plaque area $(\%)$ & $67.8(61.7 \pm 6.2)$ & $74.5(71.0 \pm 5.8)$ & $65.9(64.8 \pm 13.7)$ \\
\hline Maximum plaque thickness $(\mu \mathrm{m})$ & $515.6(493.7 \pm 121.6)$ & $540.2(566.2 \pm 56.9)$ & $463.0(475.3 \pm 56.6)$ \\
\hline Media thickness $(\mu \mathrm{m})$ & $\mathrm{I} 19.1(144.9 \pm 36.9)$ & $109.9(I I I .7 \pm 22.2)$ & $92.6(145.9 \pm 54.5)$ \\
\hline IMT & $5.07(4.3 \pm 1.2)$ & $5.58(5.87 \pm 1.4)$ & $4.99(4.31 \pm 1.5)$ \\
\hline Minimum FC thickness $(\mu \mathrm{m})$ & $29.3(34.9 \pm 12.4)$ & $54.4(55.3 \pm 11.0)$ & $51.3(48.6 \pm 3.7)$ \\
\hline Macrophage area $(\%)$ & $29.5(25.7 \pm 4.8)$ & $25.1(25.3 \pm 4.0)$ & $23.9(23.5 \pm 0.8)$ \\
\hline
\end{tabular}

Note: Data are expressed as median (mean \pm SEM).

Abbreviations: FC, fibrous cap; IMT, intima-media thickness; SEM, standard error of the mean; SPIONs, superparamagnetic iron oxide nanoparticles; SPION-DEXA, SPIONs conjugated with dexamethasone phosphate.

diet, local administration of SPION-DEXA enhanced the inflammatory burden in the developed plaques compared both with control-SPIONs and with nanoparticle-untreated atherosclerotic animals. The possible reasons for this discrepancy with previously published data are likely related

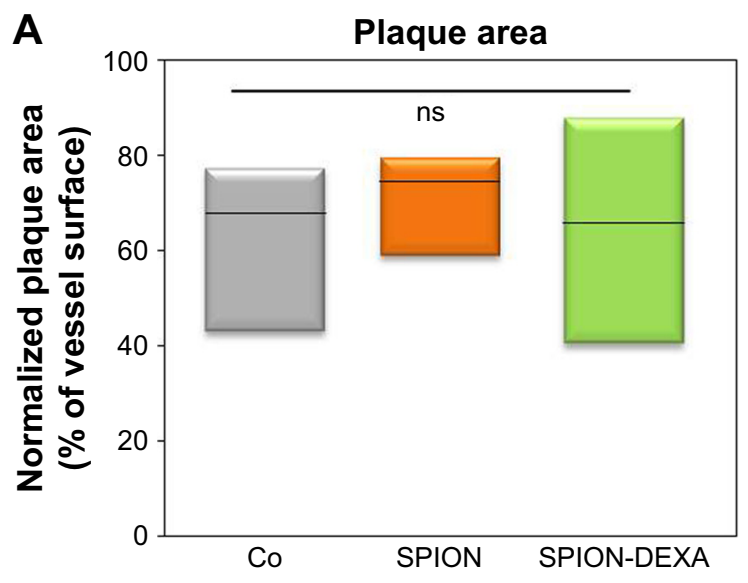

C

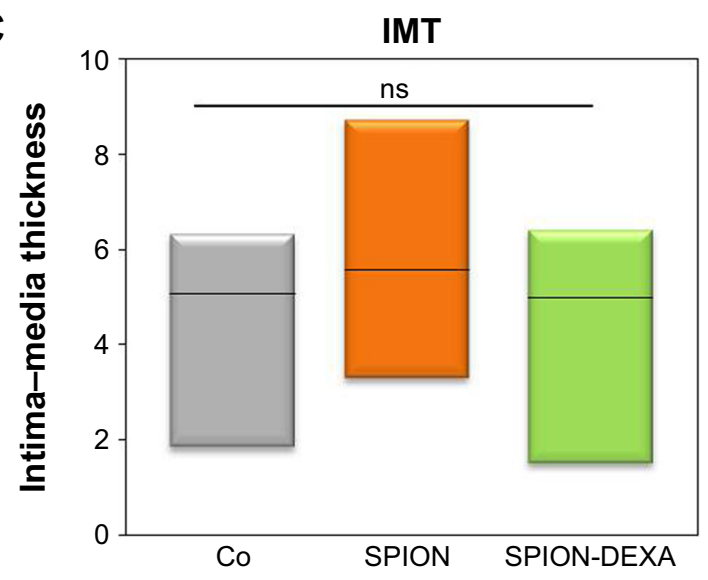

to the major differences in the study designs. The beneficial effects of DEXA were previously observed in diet-induced model of atherosclerosis, where the animals received daily intramuscular DEXA throughout the 8 weeks of diet duration, but the acute injury to the vessel was missing. ${ }^{35}$
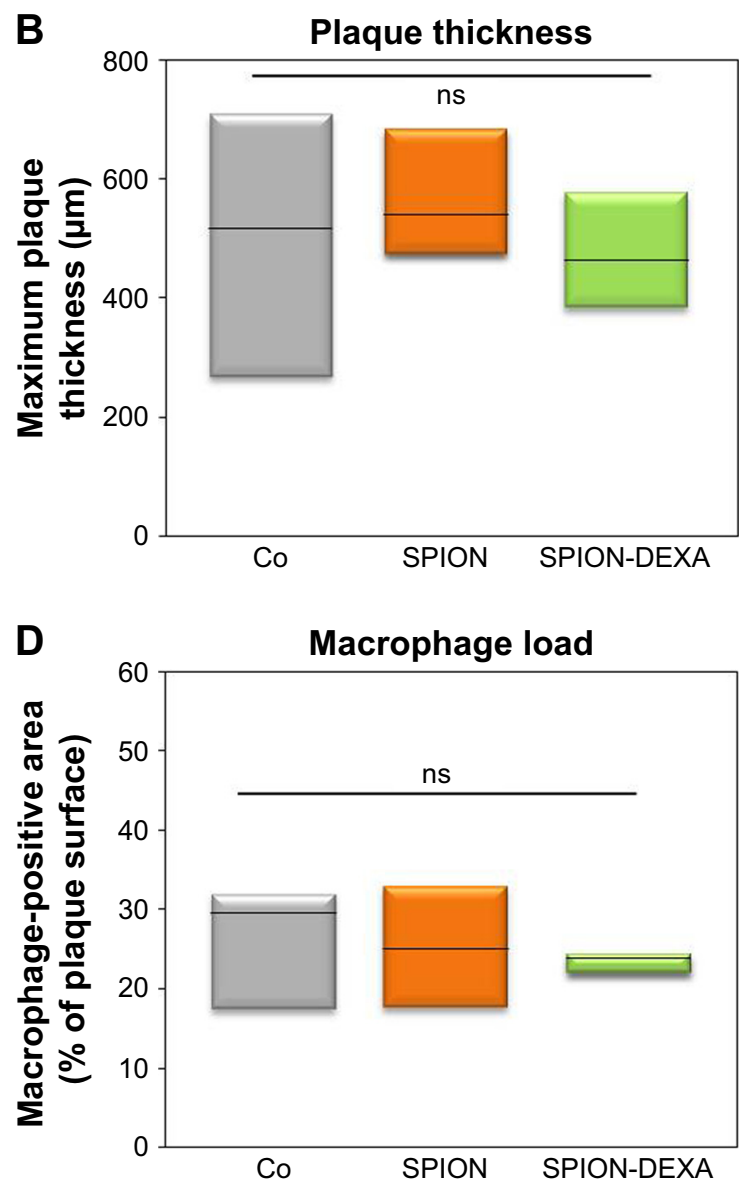

Figure 6 In vivo effects of late MDT with SPION-DEXA.

Notes: Following the balloon injury, the animals were fed high-cholesterol diet for 5 weeks and normal diet for 2 weeks. Subsequently, the animals received intra-arterial administration of either control SPION or SPION-DEXA under external magnetic field and additional 4 weeks of normal diet. Control group animals (Co) did not receive any treatment with nanoparticles. (A) Normalized plaque area; (B) maximum thickness of intima; and (C) the calculated IMT are shown. (D) Macrophage-positive area (RAM-I I staining) in animals receiving early administration of control SPION or SPION-DEXA. Graphs show median, 25th and 75th percentile.

Abbreviations: IMT, intima-media thickness; MDT, magnetic drug targeting; ns, not significant; SPION, superparamagnetic iron oxide nanoparticles; SPION-DEXA, SPIONs conjugated with dexamethasone phosphate. 
Interestingly, similar proinflammatory effects as observed in our study, including paradoxically enhanced monocyte recruitment, have been reported by van der Valk et al in low-density lipoprotein receptor knockout mice treated with liposomal prednisolone. ${ }^{36}$ The reasons for this unexpected effect of local DEXA therapy can lie in the ability of DEXA to enhance phagocytic activity of macrophages. ${ }^{37}$ In lipidrich environment, DEXA can impair cholesterol removal from vessel wall and promote the foam cell formation by enhancing the expression of ACAT-1 (acetyl-coenzyme A acetyltransferase 1), leading to increased accumulation of cholesterol esters. ${ }^{38}$ Glucocorticoid treatment may additionally delay wound healing process in the injured vessels by inhibition of endothelial nitric oxide generation ${ }^{39,40}$ and lead to less stable plaque phenotype through the suppression of smooth muscle cell migration and proliferation. ${ }^{41,42}$

In contrast to the inflammation-enhancing effect of early administration of SPION-DEXA, we did not observe any major differences between animals receiving late administration of control SPION and SPION-DEXA. In these animals, the balloon-induced endothelial injury was already healed and advanced plaques were developed at the time of administration. Interestingly, when comparing the effects of early vs late treatment with SPION-DEXA, a tendency toward stronger fibrous cap thinning (32.7 vs $51.4 \mu \mathrm{m})$ and increased macrophage-positive surface $(42 \%$ vs $24 \%$ ) was observed in early treatment group, suggesting a more vulnerable plaque. This indicates that the observed proatherogenic effect of early SPION-DEXA administration may depend on the presence of acute vascular injury.

Of note, among the tested nanoparticles and administration regimens, the most stable plaque phenotype and smallest plaque burden were observed in the animals that received early administration of control SPIONs. An anti-inflammatory effect of ultrasmall SPION administration was previously suggested in patients with myocardial infarction undergoing diagnostic MRI. ${ }^{43}$ In our study, we did not observe a reduced macrophage load in control SPION-treated animals compared with untreated group, but a slight tendency toward smaller IMT was noted. Studies in larger cohorts would be necessary to investigate the mechanisms of this process.

A limitation of this study is a relatively small number of the tested animals. Due to the unexpected results (ie, proinflammatory effect of SPION-DEXA administration), we decided to discontinue the investigations using DEXAbased approach in this model and thus limit the number of sacrificed animals. Further, it must be noted that the lateral magnetic field gradient used in this study inevitably limits the targeting efficacy to the large vessels compared with cancer microvasculature. ${ }^{6,44}$ In our previous ex vivo studies in human umbilical arteries, the magnetic accumulation of SPIONs was extensively investigated, showing that the targeting is strongly dependent on the distance between the tissue and the magnetic tip. ${ }^{17}$ Based on these studies, successful SPION accumulation along the artery distance of about $0.7-1 \mathrm{~cm}$ in both directions from the magnetic tip was expected. Further localized artery regions are not targeted using this approach. As shown in Figure 4, the accumulation of SPIONs in the region of balloon injury was achieved, but the targeting efficacy of this approach is certainly difficult to measure quantitatively. In case of early treatment when the vascular injury is acute, the unexpected proinflammatory effect of SPION-DEXA compared with control SPIONs and untreated animals seems to indicate successful delivery of at least a part of drug load. The total DEXA dose administered locally in our study was $300 \mu \mathrm{g}$, which corresponds to $0.075-0.1 \mathrm{mg} / \mathrm{kg}$ or $1.25-1.6 \mu \mathrm{g} / \mathrm{mL}$ blood. As only a single dose was given, the systemic administration would be very unlikely to produce such negative effect on the plaque development. In case of late treatment, it cannot be estimated in how far the lack of therapeutic effects was due to the drug accumulation only in limited areas. In the presence of a large advanced plaque, external targeting delivers SPIONs to a narrow area, which may strongly limit the efficacy of this approach. A possible solution to this problem could be MDT using radial magnetic field generated by MRI coil.

Taken together, we demonstrated the successful accumulation of intra-arterially administered SPIONs at the region of arterial injury in vivo, confirming the feasibility of MDT as a potential targeting approach to vascular injuries and/or atherosclerotic plaques. However, magnetic targeting of DEXA to balloon injury region had a proinflammatory effect on the subsequent plaque formation process. These data indicate that extrapolating the efficacy of (nano) drugs between different animal models and from animal model to humans is extremely challenging and requires in vivo demonstration.

Despite the fact that SPION-DEXA application to vascular injury in the presence of cholesterol loading did not produce the expected anti-inflammatory results, our in vitro data pointed to good biocompatibility and targeting efficacy of these particles. SPION-DEXA may therefore be potentially suitable for local targeting of inflammatory or autoimmune diseases including gout or rheumatoid arthritis. However, for an efficient treatment of atherosclerotic plaques by MDT, SPIONs loaded with other drug candidates, eg, statins, must be tested in the future studies. 


\section{Acknowledgments}

The authors thank Dr Marina Pöttler, Bianca Weigel, and Julia Band for help with animal experiments, Eveline Schreiber for help with nanoparticle preparation, and Hatice Genç for performing nanoparticle uptake experiments. Further, we thank Prof. Dr med. Matthias Beckmann (Department of Gynaecology, University Hospital Erlangen, Germany) for providing umbilical cords, as well as Heike Kloos and Doris Flick (Laboratory of Molecular Cardiology, University Hospital Erlangen, Germany) for help with HUVEC isolation. The present work was performed in partial fulfillment of the requirements for obtaining the degree "Dr Med" by BL at the Friedrich-Alexander Universität Erlangen-Nürnberg (FAU), Germany. This work was funded by the Deutsche Forschungsgemeinschaft (German Research Foundation; CI 162/2-1, CI 162/2-3) and in part by the European Union "NanoAthero" project. The support from Manfred Roth Foundation, Fürth and from the Friedrich-Alexander-Universität Erlangen-Nürnberg (FAU) within the funding program Open Access Publishing is acknowledged.

\section{Disclosure}

The authors report no conflicts of interest in this work.

\section{References}

1. Lozano R, Naghavi M, Foreman K, et al. Global and regional mortality from 235 causes of death xfor 20 age groups in 1990 and 2010: a systematic analysis for the Global Burden of Disease Study 2010. Lancet. 2012;380(9859):2095-2128.

2. Libby P, Theroux P. Pathophysiology of coronary artery disease. Circulation. 2005;111(25):3481-3488.

3. Cicha I, Garlichs CD, Alexiou C. Cardiovascular therapy through nanotechnology - how far are we still from bedside? Eur J Nanomed. 2014;6(2):63-87.

4. Thorat ND, Bohara RA, Yadav HM, Tofail SAM. Multi-modal MR imaging and magnetic hyperthermia study of $\mathrm{Gd}$ doped $\mathrm{Fe}_{3} \mathrm{O}_{4}$ nanoparticles for integrative cancer therapy. RSC Adv. 2016;6(97):94967-94975.

5. Lyer S, Tietze R, Jurgons R, et al. Visualisation of tumour regression after local chemotherapy with magnetic nanoparticles - a pilot study. Anticancer Res. 2010;30(5):1553-1557.

6. Tietze R, Lyer S, Dürr S, et al. Efficient drug-delivery using magnetic nanoparticles - biodistribution and therapeutic effects in tumour bearing rabbits. Nanomedicine. 2013;9(7):961-971.

7. Janko C, Dürr S, Munoz LE, et al. Magnetic drug targeting reduces the chemotherapeutic burden on circulating leukocytes. Int J Mol Sci. 2013;14(4):7341-7355.

8. Ma YH, Wu SY, Wu T, Chang YJ, Hua MY, Chen JP. Magnetically targeted thrombolysis with recombinant tissue plasminogen activator bound to polyacrylic acid-coated nanoparticles. Biomaterials. 2009; 30(19):3343-3351.

9. Zhang Y, Li W, Ou L, et al. Targeted delivery of human VEGF gene via complexes of magnetic nanoparticle-adenoviral vectors enhanced cardiac regeneration. PLoS One. 2012;7(7):e39490.

10. Chao X, Zhang Z, Guo L, et al. A novel magnetic nanoparticle drug carrier for enhanced cancer chemotherapy. PLoS One. 2012;7(10):e40388.

11. Elbialy NS, Fathy MM, Khalil WM. Doxorubicin loaded magnetic gold nanoparticles for in vivo targeted drug delivery. Int J Pharm. 2015; 490(1-2):190-199.
12. Yu J, Ju Y, Zhao L, et al. Multistimuli-regulated photochemothermal cancer therapy remotely controlled via $\mathrm{Fe}_{5} \mathrm{C}_{2}$ nanoparticles. ACS Nano. 2016;10(1):159-169.

13. Byrne JD, Betancourt T, Brannon-Peppas L. Active targeting schemes for nanoparticle systems in cancer therapeutics. Adv Drug Deliv Rev. 2008;60(15):1615-1626.

14. Ma HL, Qi XR, Ding WX, Maitani Y, Nagai T. Magnetic targeting after femoral artery administration and biocompatibility assessment of superparamagnetic iron oxide nanoparticles. $J$ Biomed Mater Res A. 2008;84(3):598-606.

15. Chen H, Kaminski MD, Pytel P, Macdonald L, Rosengart AJ. Capture of magnetic carriers within large arteries using external magnetic fields. J Drug Target. 2008;16(4):262-268.

16. Richter H, Wiekhorst F, Schwarz K, et al. Magnetorelaxometric quantification of magnetic nanoparticles in an artery model after ex vivo magnetic drug targeting. Phys Med Biol. 2009;54(18):N417-N424.

17. Janikowska A, Matuszak J, Lyer S, et al. A novel human artery model to assess the magnetic accumulation of SPIONs under flow conditions. Sci Rep. 2017;7:42314.

18. Tietze R, Rahn H, Lyer S, et al. Visualization of superparamagnetic nanoparticles in vascular tissue using $\mathrm{X} \mu \mathrm{CT}$ and histology. Histochem Cell Biol. 2011;135(2):153-158.

19. Polyak B, Medved M, Lazareva N, et al. Magnetic nanoparticlemediated targeting of cell therapy reduces in-stent stenosis in injured arteries. ACS Nano. 2016;10(10):9559-9569.

20. Flammer JR, Rogatsky I. Minireview: Glucocorticoids in autoimmunity: unexpected targets and mechanisms. Mol Endocrinol. 2011;25(7): 1075-1086.

21. Ji JY, Jing H, Diamond SL. Shear stress causes nuclear localization of endothelial glucocorticoid receptor and expression from the GRE promoter. Circ Res. 2003;92(3):279-285.

22. Lobatto ME, Calcagno C, Otten MJ, et al. Pharmaceutical development and preclinical evaluation of a GMP-grade anti-inflammatory nanotherapy. Nanomedicine. 2015;11(5):1133-1140.

23. Hadoke PW, Iqbal J, Walker BR. Therapeutic manipulation of glucocorticoid metabolism in cardiovascular disease. Br J Pharmacol. 2009; 156(5):689-712.

24. Hämäläinen M, Nieminen R, Uurto I, et al. Dexamethasone-eluting vascular stents. Basic Clin Pharmacol Toxicol. 2013;112(5):296-301.

25. Ribichini F, Tomai F, Paloscia L, et al. Steroid-eluting stents in patients with acute coronary syndrome: the dexamethasone eluting stent Italian registry. Heart. 2007;93(5):598-600.

26. Hoffmann R, Radke PW, Ortlepp JR, et al. Intravascular ultrasonic comparative analysis of degree of intimal hyperplasia produced by four different stents in the coronary arteries. Am J Cardiol. 2004; 94(12):1548-1550.

27. Han SH, Ahn TH, Kang WC, et al. The favorable clinical and angiographic outcomes of a high-dose dexamethasone-eluting stent: randomized controlled prospective study. Am Heart J. 2006;152(5): 887.e1-887.e7.

28. König A, Leibig M, Rieber J, et al. Randomized comparison of dexamethasone-eluting stents with bare metal stent implantation in patients with acute coronary syndrome: serial angiographic and sonographic analysis. Am Heart J. 2007;153(6):979.e1-979.e8.

29. Park YM, Han SH, Lee K, et al. Dexamethasone-eluting stents had sustained favorable ischemic driven target lesion revascularization rates over 5 years: a randomized controlled prospective study. Int J Cardiol. 2013;165(2):359-362.

30. Reil TD, Sarkar R, Kashyap VS, Sarkar M, Gelabert HA. Dexamethasone suppresses vascular smooth muscle cell proliferation. J Surg Res. 1999;85(1):109-114.

31. Zaloga J, Janko C, Nowak J, et al. Development of a lauric acid/albumin hybrid iron oxide nanoparticle system with improved biocompatibility. Int J Nanomedicine. 2014;9:4847-4866.

32. Matuszak J, Baumgartner J, Zaloga J, et al. Nanoparticles for intravascular applications: physicochemical characterization and cytotoxicity testing. Nanomedicine. 2016;11(6):597-616. 
33. Buechler C, Ritter M, Orsó E, Langmann T, Klucken J, Schmitz G. Regulation of scavenger receptor CD163 expression in human monocytes and macrophages by pro- and antiinflammatory stimuli. J Leukoc Biol. 2000;67(1):97-103.

34. Gordon D, Kobernick SD, Mcmillan GC, Duff GL. The effect of cortisone on the serum lipids and on the development of experimental cholesterol atherosclerosis in the rabbit. J Exp Med. 1954;99(4):371-386.

35. Naito M, Yasue M, Asai K, et al. Effects of dexamethasone on experimental atherosclerosis in cholesterol-fed rabbits. J Nutr Sci Vitaminol. 1992;38(3):255-264.

36. van der Valk FM, Schulte DM, Meiler S, et al. Liposomal prednisolone promotes macrophage lipotoxicity in experimental atherosclerosis. Nanomedicine. 2016;12(6):1463-1470.

37. Mccoll A, Michlewska S, Dransfield I, Rossi AG. Effects of glucocorticoids on apoptosis and clearance of apoptotic cells. Sci World J.2007; 7:1165-1181.

38. Yang L, Yang JB, Chen J, et al. Enhancement of human ACAT1 gene expression to promote the macrophage-derived foam cell formation by dexamethasone. Cell Res. 2004;14(4):315-323.

39. Johns DG, Dorrance AM, Tramontini NL, Webb RC. Glucocorticoids inhibit tetrahydrobiopterin-dependent endothelial function. Exp Biol Med. 2001;226(1):27-31.
40. Iuchi T, Akaike M, Mitsui T, et al. Glucocorticoid excess induces superoxide production in vascular endothelial cells and elicits vascular endothelial dysfunction. Circ Res. 2003;92(1):81-87.

41. Voisard R, Seitzer U, Baur R, et al. Corticosteroid agents inhibit proliferation of smooth muscle cells from human atherosclerotic arteries in vitro. Int J Cardiol. 1994;43(3):257-267.

42. van Put DJ, van Hove CE, de Meyer GR, Wuyts F, Herman AG, Bult H. Dexamethasone influences intimal thickening and vascular reactivity in the rabbit collared carotid artery. Eur J Pharmacol. 1995;294(2-3): $753-761$.

43. Florian A, Ludwig A, Rösch S, et al. Positive effect of intravenous ironoxide administration on left ventricular remodelling in patients with acute ST-elevation myocardial infarction - a cardiovascular magnetic resonance (CMR) study. Int J Cardiol. 2014;173(2):184-189.

44. Thorat ND, Bohara RA, Noor MR, Dhamecha D, Soulimane T, Tofail SAM. Effective cancer theranostics with polymer encapsulated superparamagnetic nanoparticles: combined effects of magnetic hyperthermia and controlled drug release. ACS Biomater Sci Eng. 2017;3(7): $1332-1340$. 


\section{Supplementary materials}

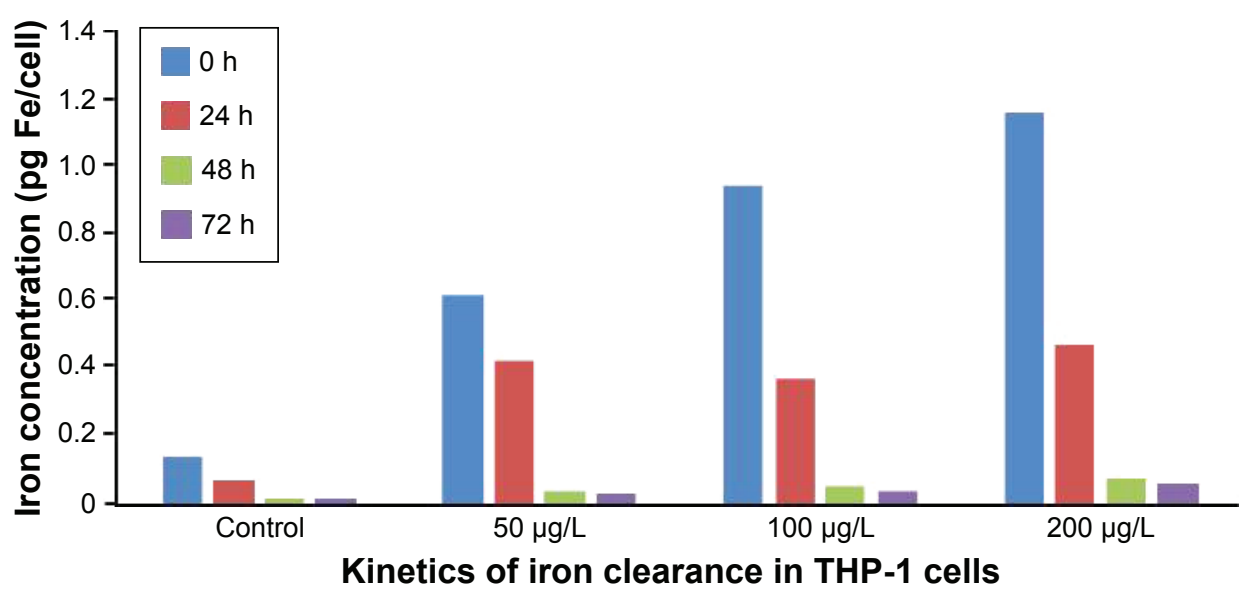

Figure SI In vitro uptake and clearance of SPIONs in THP-I monocytic cells.

Notes: THP-I cells (triplicate samples) were incubated with given concentrations of SPIONs for 24 hours. Afterward, the cells were washed to remove noninternalized SPIONs and cultured for further 72 hours in the absence of nanoparticles. The measurement of iron content per cell was done using atomic emission spectroscopy at the time of SPION removal ( 0 hour) and after 24, 48, and 72 hours postremoval. Gradual decrease in cellular iron content is observed over 72 hours due to cell metabolism and proliferation.

Abbreviation: SPIONs, superparamagnetic iron oxide nanoparticles. 

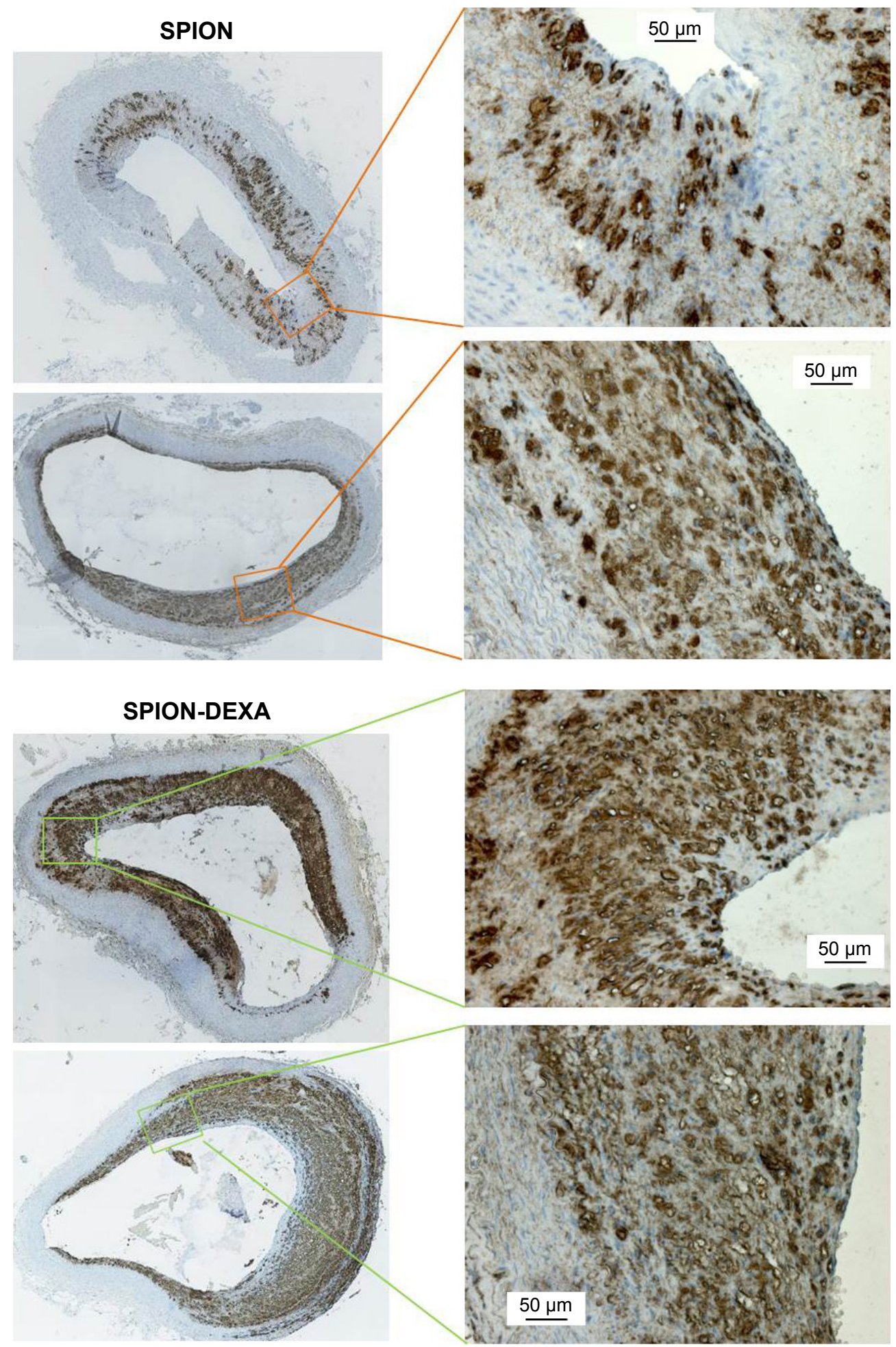

Figure S2 In vivo effects of early MDT with SPION-DEXA on macrophage load.

Notes: Following the balloon injury, the animals were intraarterially administered either control SPION $(n=5)$ or SPION-DEXA ( $n=4)$ under external magnetic field. After 5 weeks of high-cholesterol diet and normal diet for 2 weeks, animals were sacrificed and the excised aortas analyzed histochemically. Mouse monoclonal antibody against rabbit macrophages (RAM-1 I, I:50 dilution) was used to detect plaque macrophages. Digital images of the plaques at $\times 10$ objective magnification (left, overview images) or 20x objective magnification (right) were obtained using AxioObserver.ZI microscope. The representative example images from two different animals per each group show macrophage accumulation in the atherosclerotic plaques.

Abbreviations: MDT, magnetic drug targeting; SPION, superparamagnetic iron oxide nanoparticle; SPION-DEXA, SPIONs conjugated with dexamethasone phosphate; THP-I, a human monocytic cell line. 


\section{Publish your work in this journal}

The International Journal of Nanomedicine is an international, peerreviewed journal focusing on the application of nanotechnology in diagnostics, therapeutics, and drug delivery systems throughout the biomedical field. This journal is indexed on PubMed Central,

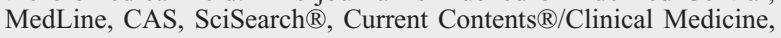

Journal Citation Reports/Science Edition, EMBase, Scopus and the Elsevier Bibliographic databases. The manuscript management system is completely online and includes a very quick and fair peer-review system, which is all easy to use. Visit http://www.dovepress.com/ testimonials.php to read real quotes from published authors.

Submit your manuscript here: http://www.dovepress.com/international-journal-of-nanomedicine-journal 